\title{
Mechanisims of asthma and allergic disease - 1085. Safety and tolerability of escalating doses of house dust mite peptide antigen desensitisation
}

\author{
Roderick Peter Hafner ${ }^{1 *}$, Mark Larché ${ }^{2}$, Paul Laidler ${ }^{1}$, Pascal Hickey ${ }^{3}$, Jacques Hébert ${ }^{4}$ \\ From 2nd WAO International Scientific Conference (WISC 2012) \\ Hyderabad, India. 6-9 December 2012
}

\section{Background}

House Dust Mite (HDM) accounts for 20-25\% of the allergic rhinoconjunctivitis disease burden worldwide. Previous studies have shown immunotherapy using peptides from Fel $\mathrm{d} 1$ can induce tolerance in cat allergic subjects. This study identified T-cell epitopes derived from HDM allergens and evaluated their safety and efficacy in a clinical study.

\section{Methods}

Potential T-cell epitopes were identified by algorithm, screened for ability to stimulate $\mathrm{T}$-cell responses in exvivo blood samples from HDM allergic subjects and tested to confirm they did not cause basophil histamine release. A second group of HDM allergic subjects attended a challenge where Conjunctival Provocation Test (CPT) response and Early (EPSR) and Late Phase Skin Response (LPSR) were measured. Subjects were randomised to one of 5 cohorts of 10 subjects. In each cohort 8 subjects received HDM peptide antigen desensitisation (PAD) using the identified T-cell epitope mixture and 2 subjects received placebo. The first cohort received $4 \times 0.03 \mathrm{nmol}$ 4weeks(wk) apart; successive cohorts received 4 administrations 4 wk apart of $0.3,1,3$ and $12 \mathrm{nmol}$, respectively. EPSR, LPSR and CPT were re-measured 18-22wk after starting treatment.

\section{Results}

HDM-PAD was safe and well tolerated with no Serious Adverse Events. The largest number of Treatment Emergent Adverse Events (TEAEs) occurred in the 0.03nmol group and the least in the $3 \mathrm{nmol}$ group. The most commonly reported TEAEs in subjects who received HDM-PAD were nasopharyngitis, influenza, gastroenteritis and nausea. There were no changes in mean FEV1 on dosing days for any dose of HDM-PAD or placebo. Subjects treated with four of the five HDM-PAD doses showed changes from baseline in CPT score at 18-22wk of between $-16.7 \%$ to $-41.4 \%$, compared with no change for placebo. A statistically significant median \%change from baseline in CPT score of $-36.7 \%$ ( $\mathrm{p}=0.0257$ vsplacebo) and the largest change in EPSR (median \%change -39.19\%) and LPSR (median \%change -51.19\%) was observed after 3nmol HDM-PAD.

\section{Conclusions}

HDM-PAD is safe and well tolerated when given as 4 intradermal injections $4 \mathrm{wk}$ apart, at doses up to $12 \mathrm{nmol}$ in HDM allergic subjects. Reductions in EPSR, LPSR and CPT after HDM-PAD indicate the identified T-cell epitopes have biological activity and merit further evaluation for treatment of HDM allergy.

\section{Author details \\ ${ }^{1}$ Circassia Limited, Oxford, UK. ${ }^{2}$ Department of Medicine, Mcmaster University, Hamilton, ON, Canada. ${ }^{3}$ Adiga Life Sciences Inc., Hamilton, ON, Canada. ${ }^{4}$ Centre De Recherche Appliqué En Allergie De Québec, Québec City, QC, Canada.}

Published: 23 April 2013

\section{doi:10.1186/1939-4551-6-S1-P81}

Cite this article as: Hafner et al:: Mechanisims of asthma and allergic disease -1085 . Safety and tolerability of escalating doses of house dust mite peptide antigen desensitisation. World Allergy Organization Journal 2013 6(Suppl 1):P81.

${ }^{1}$ Circassia Limited, Oxford, UK

Full list of author information is available at the end of the article

(C) 2013 Hafner et al; licensee BioMed Central Ltd. This is an Open Access article distributed under the terms of the Creative Commons Attribution License (http://creativecommons.org/licenses/by/2.0), which permits unrestricted use, distribution, and reproduction in any medium, provided the original work is properly cited. 